\title{
Research on the Energy-Efficient Caching Strategy in Content-Centric Networking
}

Tiantian Shi

School of Communication and Information Engineering, Chongqing University of posts and telecommunications, Chongqing, China,

E-mail: $\underline{\text { stt0802@126.com }}$

\author{
Li Zhang \\ School of Communication and Information Engineering, \\ Chongqing University of posts and telecommunications, \\ Chongqing, China, \\ E-mail:243666882@qq.com
}

\author{
Changbiao $\mathrm{Xu}$ \\ College of Photo electronic Engineering, \\ Chongqing University of posts and telecommunications, \\ Chongqing, China, \\ E-mail: xucb@,cqupt.edu.cn
}

applications and are only suitable for particular topology types (e.g, hierarchical, adaptive overlay structures). The default cache strategy in CCN is LCE (Leave Copy Everywhere). LCD (Leave Copy Down) [4] and MCD (move copy down) [5] are simple caching strategies which have not consider the energy strategies form ISPs. The problem of energy efficient in-network caching in $\mathrm{CCN}$ is studied in [6].Then the energy consumption model for content distribution is built and an aging popularity-based in-network caching scheme is proposed to reach the energy efficiency. In [7], the authors investigate the problem of caching strategy about chunk placement in $\mathrm{CCN}$, and a caching location and searching scheme (CLS) in CCN hierarchical infrastructure is proposed. Although much work has been done in $\mathrm{CCN}$, however, the problem of content caching to optimize the whole network performance in $\mathrm{CCN}$ is not well considered. However, the caching optimization problem in $\mathrm{CCN}$ is one of the most important issues to balance the network load, save the energy consumption and achieve the green communication.

Therefore, an energy-efficient caching strategy (EECS) in Content-Centric Networking is proposed in this paper. By monetary consumption and router revenue to design a reasonable cache threshold, the data which has higher popularity and much higher energy-efficient will be cached first. In addition to, a cost-performance ratio (CPR) algorithm is proposed to replace the content when the cache space is full. Thus, the network gets low service delay and communication energy, also the promotion of availability and efficiency can be achieved. Simulation results show that the strategy can effectively reduce network overhead and delay, and obtain better performance.

\section{CONTENT-CENTRIC NetwORKING}

In the $\mathrm{CCN}$ network, users don't care about the location of the content, but the content itself. Network can unified identify the content, then location, routing and transmission based on the content, the user get the content by sending the interest packet which carries content request information, content providers provide the 
corresponding data packet according to the request information. In addition, $\mathrm{CCN}$ nodes can cache the content which can speed up the efficiency of other users access network, reduce network congestion status, improve the utilization rate of network resources. Caching mechanism has been one of hot topics in $\mathrm{CCN}$ research field.

\section{A. Packet types}

Fig .1 shows that $\mathrm{CCN}$ consists of Interest packet and Data packet. Interest packet carries a name that identifies the desired data. Once the Interest reaches a node that has the requested data, a Data packet is sent back, which carries both the name and the content of the data, together with a signature by the producer's key. This Data packet traces in reverse the path created by the Interest packet back to the consumer. Neither Interest nor Data packets carry any host or interface addresses; Interest packets are routed towards data producers based on the names carried in the Interest packets, and Data packets are returned based on the state information set up by the Interests at each router hop.
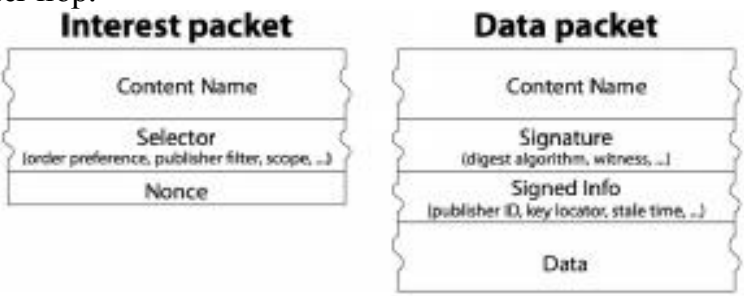

Figure 1.Packets in the $\mathrm{CCN}$

\section{B. Architecture of CCN nodes}

$\mathrm{CCN}$ nodes include routers and end nodes, mainly through three elements to implement the packet forwarding mechanism and network storage function. They are respectively Content storage (CS), the Pending Interest Table (PIT) and Forwarding Information Base (FIB).

\section{Packet forwarding mechanism}

When an Interest packet arrives at a $\mathrm{CCN}$ face, the Content Name carried by this Interest is firstly checked in the CS. If there is a matching Data, it will be directly returned through the same face where the Interest arrived at. Otherwise the Content Name is further checked in PIT. If there is already a matching entry, the arrival face information is updated in the matching entry. If not, the FIB table is consulted for the forwarding information, and a new entry associated with the new arrival Interest and its incoming face is created in PIT.

When a Data packet arrives at a $\mathrm{CCN}$ node, the Content Name of the Data is checked in the CS. If there is already a matching content cached in the CS, this Data packet should be discarded. If there is no existing match in CS, the Data is checked in PIT. A PIT entry match means this Data has been required, it is sent out through the list of faces associated with the matching PIT entry, and the Data can be stored in the CS.

\section{ENERGY-EFFICIENT CACHING STRATEGY FOR CONTENT-CENTRIC NETWORKING}

Content caching in $\mathrm{CCN}$ can be coordinated or noncoordinated, similar to that in the Internet [8]. Noncoordinated caching mechanisms store only the locally most popular contents at each $\mathrm{CCN}$ router, without coordination with other routers. Non-coordinated caching mechanisms store is used in this paper. The related model definition as follows:

Topology model: CCN network is supported by the underlying infrastructures can be viewed as a connection diagram $G=(V, E)$, where $V=\left\{v_{1}, v_{2}, \ldots, v_{n}\right\}$ is the set of network node and the node is just the router, $E$ is the set of link, $e=\left(v_{i}, v_{j}\right) \in E$, where $\left(v_{i}, v_{j}\right) \in E$ shows that node $v_{i}$ and node $v_{j}$ are connected. Each node $v \in V$ provides caching and computing resources and the capacity of each node is $C_{i}$, each link provides bandwidth resource. All of these resources are limited in the CCN.

Content model: suppose a directory, there are $m$ contents, $S=\left(s_{1}, s_{2}, \ldots, s_{m}\right)$ and where $S=\sum_{k=1}^{m} s_{k}$.

\section{A. Problem definition}

Definition 1 Design the cache threshold $f_{i k}$.Assuming that $T_{0}(k)$ is the starting time that the content $k$ via the node $v_{i}$ for the first time, $T_{1}(k)$ is the time that content $k$ via the node $v_{i}$ for the number of $f_{i k}, f_{i k}$ is he total number of content $k$ via the node $v_{i}$ form $T_{0}(k)$ to $T_{1}(k)$.

Energy-efficient model is that the node decides whether store the content when router revenue is higher than the cache consumption.

Form $T_{0}(k)$ to $T_{1}(k)$, cache consumption $C_{i k}$ is defined as (1).

$$
C_{i k}=D_{k} \cdot\left[T_{1}(k)-T_{0}(k)\right] \cdot P_{i c}
$$

The router revenue $S_{i k}$ is defined as (2).

$$
S_{i k}=f_{i k} \cdot P_{i k}
$$

Where $C_{i k}$ is the total consumption that router $v_{i}$ caches content $k$ form $T_{0}(k)$ to $T_{1}(k), S_{i k}$ is the revenue that router caches content $k, D_{k}$ is the size[bits] of the content $k, P_{i c}$ is the consumption $[\$ /(\mathrm{bit} \cdot \mathrm{s})]$ that the router caches the content $k . P_{i k}$ is the router revenue that the user downloads the content $k$ form the router each time. When $S_{i k} \geq C_{i k}$, the router decides whether store the content $k$, the detail as follows.

$$
\begin{gathered}
f_{i k} \cdot P_{i k} \geq D_{k} \cdot\left[T_{1}(k)-T_{0}(k)\right] \cdot P_{i c} \\
f_{i k} \geq \frac{D_{k} \cdot\left[T_{1}(k)-T_{0}(k)\right] \cdot P_{i c}}{P_{i k}}
\end{gathered}
$$

For the content $k$, When $f_{i k}$ meets (4), the router 
will store the content $k$, otherwise, the router does not store the content $k$.

Definition 2 Different routers have different cache mediums, so the cache consumption is different. In this paper, suppose the routers use the DRAM and the years of use are 5 years. So $P_{i c}$ is $7.74 * 10^{-16}[\$ /($ bit $\cdot \mathrm{s})]$.

Definition $3 P_{i k}$ is the router revenue that the user downloads the content $k$ form the router each time. In this paper, the contents mainly include Web、 audio、 video and so on. Where the price of web is 0 , that is to say, the router revenue is 0 that the user obtains the web content form the router. The price of the rest content is random.

Definition 4 Assuming that the time of router $v_{i}$ caches content $k$ is $T_{i c}(k)$, which is defined as (5).

$$
T_{i c}(k)=T_{i r}(k)-T_{i s}(k)
$$

The cost-performance ratio (CPR) $R_{i k}$ of the cache content is defined as (6).

$$
R_{i k}=\frac{N_{i k} \cdot P_{i k}}{D_{k} \cdot\left[T_{i r}(k)-T_{i s}(k)\right]}
$$

The router $v_{i}$ has cached the content $k(k=1,2, \ldots$, $m), T_{i s}(k)$ is the initial time of the content $k$ is cached by router $v_{i}, T_{i r}(k)$ is the current system time. $N_{i k}$ is the number of content $k$ is downloaded during the time $T_{i c}(k)$. Comparing the cost-performance ratio $R_{i k}$ of the content $k$, when the router $v_{i}$ cache space is full, the content $v_{i}$ will replace the content $k$ which the $R_{i k}$ is the minimum.

\section{B. The steps of energy-efficient caching strategy}

- Step1: When the content $k$ arrives to a router, the router gets the information of content $k$ and determines whether content $k$ arrives the router for the first time, and if so, the router records the time $T_{0}(k)$ and the number $f_{i k}=1$, if not, the router updates the number $f_{i k}$ that the content via the router. Then the router judges whether $f_{i k}$ meets the energy-efficient model.

- Step2: If $f_{i k}$ meets the energy-efficient model, the router stores the content $k$. At this time, if the router has cache space, the router stores the content $k$, if the cache space of router is full, the router performs the CPR replacement algorithm and selects the content which the cost-performance ratio $R_{i k}$ is the minimum. At last, the content $k$ replaces of the content which is substituted.
- Step3: If $f_{i k}$ isn't meet the energy-efficient model, the router updates the number $f_{i k}$. In this paper, the size of the content is the same.

\section{Simulation EVAluation}

In this paper, the simulation is based on the ndnSIM of NS-3 under Linux environment, Fig .2 is the network topology. Service nodes provide 500 different contents, each content size is $1024 \mathrm{~KB}$. The content popularity is a very important factor of influencing the CCN storage performance, the most typical data popularity is Zipf distribution [9], the distribution shows the different states of popularity distribution by the change of parameters $\alpha$ $(\geq 0)$, the more $\alpha$ is close to 0 , the smaller the popularity gap among contents, on the contrary, $\alpha$ is larger, the popularity gap among contents is bigger. Normally, $\alpha$ value is between 0.6 and 1.2 [10], therefore, the content's popularity follows Zipf distribution and $\alpha$ is 0.8 in this article.

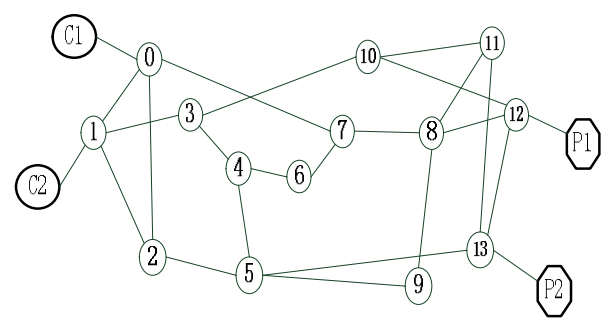

Figure 2.NSF Network

The request packets of users $\mathrm{C} 1$ and $\mathrm{C} 2$ enter the network by edge nodes 0 and 1 , request frequency follows Poisson Distribution $\lambda=10 \mathrm{req} / \mathrm{s}$, Service nodes P1 and P2 respectively connect with node 12 and node 13 which provide the users with corresponding request packets. Each In-network node has limited Content Store (CS). The total capacity of CS is $10 \%-50 \%$ of the total content size. Each CS performs CPR replacement strategy. Comparison algorithms are LCE and Prob (p) storage strategies, simulation time is $300 \mathrm{sec}$.

We now compare three caching policies in view of the average hit ratio in the network. Fig . 3 shows the average hit ratio of user requests within the network, which is under different in-network storage conditions. The hit ratio is requests of all the service nodes divided by the total number of requests in each sampling instant. Compared with the other two strategies, EECS strategy improves the hit ratio of the request in the network. For example, when network storage capacity occupies $40 \%$ of the total content size, the average hit ratios of three strategies are $31.8 \%, 28.9 \%, 27.6 \%$. Because that the router with EECS strategy can cache the content which is requested with much more times.

Fig .4 shows the average hit length under each scheme, where the hit length is measured in number hops from the client to the hit cache. The lower the average hit length, the better the performance. The average hit length in EECS strategy is lower than that in the other policies. Fig .5 shows the average delay of the client obtains content, the value is the average time of all nodes obtain content. The average delay in EECS strategy is lower than that in the other policies. By using the EECS strategy proposed in this paper, the nodes can cache more different 
contents which improve the content diversity. The download time is reduced in EECS strategy over the other strategies.

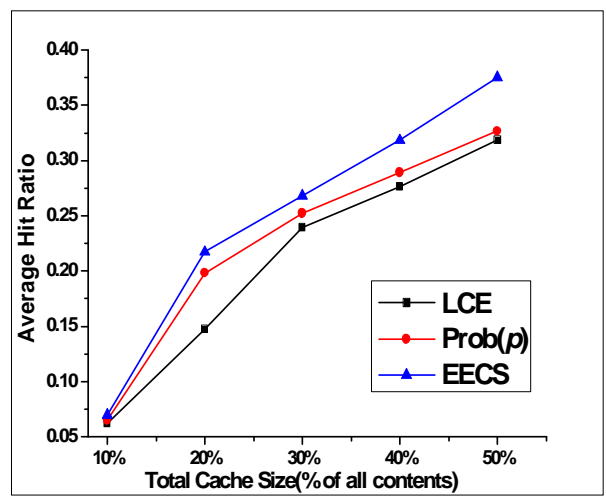

Figure 3.Average hit ratio

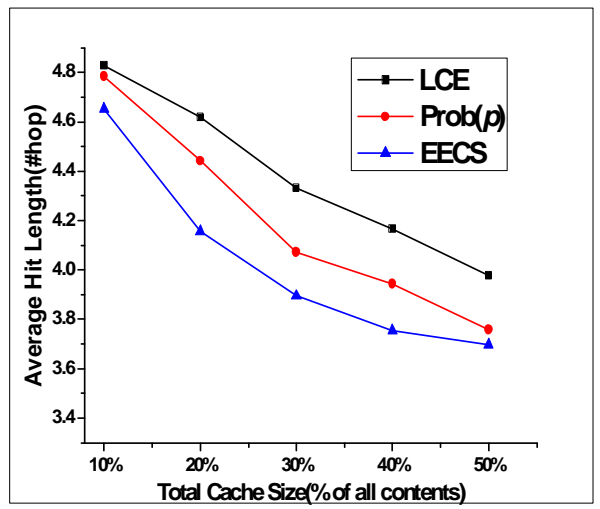

Figure 4.Average hit length

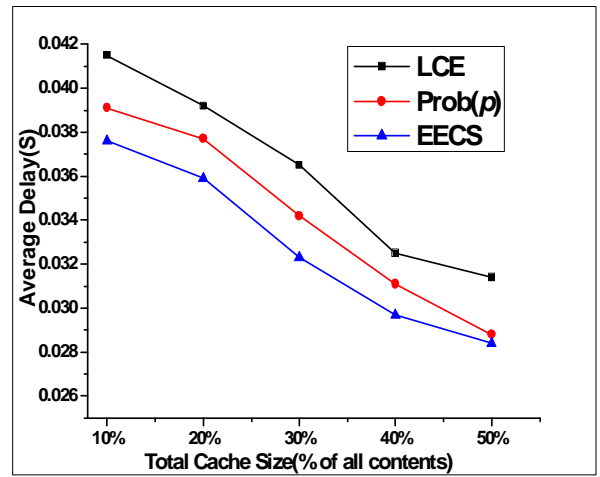

Figure 5.Average Delay (s)

Fig .6 shows the average bandwidth consumption required to satisfy a request. From the figures, it can be clearly seen that the EECS strategy results in much lower load on the network links than the other strategies. That is because EECS just cache the contents which are beneficial to the nodes. These results indicate that the threshold-based caching can use network resources such as memory usage and bandwidth.

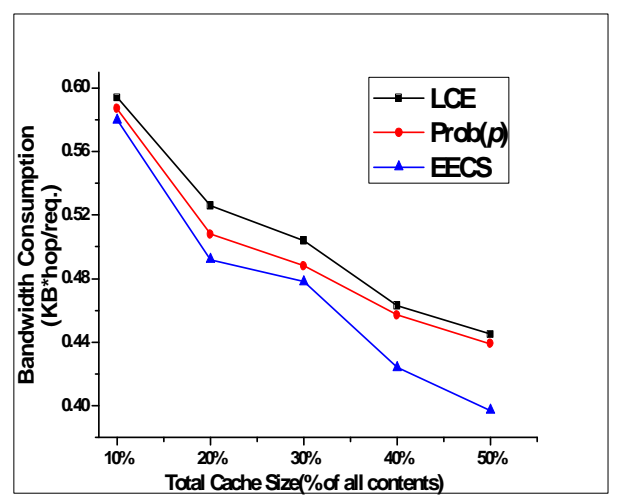

Figure 6.Bandwidth Consumption

\section{CONCLUSIONS}

In this paper, we consider a game between an Internet Service (access) Provider (ISP) and content provider (CP) on a platform of end-user demand. The energy-efficient non-cooperative caching strategy and replacement algorithm of the cost-performance ratio (CPR) in contentcentric networking are proposed. Simulation results show that the strategy can effectively reduce network overhead and delay, and obtain better performance. As future work, we will further evaluate energy-efficient of the proposed mechanism under the different total size of CSs and we also plan to propose an energy-efficient cooperative cache strategy. Then the routers use different caching strategies to store contents under different situations to obtain better performance and improve the resource utilization.

\section{REFERENCES}

[1] Ghodsi A, Shenker S, Koponen T, et al. Information-centric networking: seeing the forest for the trees[C]//Proceedings of the 10th ACM Workshop on Hot Topics in Networks. ACM, 2011: 1.

[2] Zhang L, Estrin D, Burke J, et al. Named Data Networking (NDN) Project $[\mathrm{J}]$. Relatório Técnico NDN-0001, Xerox Palo Alto Research Center-PARC, 2010.

[3] Laoutaris N, Zissimopoulos V, Stavrakakis I. On the optimization of storage capacity allocation for content distribution[J]. Computer Networks, 2005, 47(3): 409-428.

[4] Laoutaris N, Che H, Stavrakakis I. The LCD interconnection of LRU caches and its analysis [J]. Performance Evaluation, 2006, 63(7): 609-634.

[5] Laoutaris N, Syntila S, Stavrakakis I. Meta algorithms for hierarchical web caches[C]//Performance, Computing, and Communications, 2004 IEEE International Conference on. IEEE, 2004: 445-452.

[6] Li J, Liu B, Wu H. Energy-efficient in-network caching for content-centric networking [J]. Communications Letters, IEEE, 2013, 17(4): 797-800.

[7] Li Y, Lin T, Tang H, et al. A chunk caching location and searching scheme in content centric networking [C]// Communications (ICC), 2012 IEEE International Conference on. IEEE, 2012: 2655-2659.

[8] Borst S, Gupta V, Walid A. Distributed caching algorithms for content distribution networks[C]//NFOCOM, 2010 Proceedings IEEE. IEEE, 2010: 1-9.

[9] Carofiglio G, Gallo M, Muscariello L, et al. Modeling data transfer in content-centric networking[C]//Proceedings of the $23 \mathrm{rd}$ International Teletraffic Congress. International Teletraffic Congress, 2011: 111-118.

[10] Rosensweig E J, Kurose J, Towsley D. Approximate models for general cache networks[C]//INFOCOM, 2010 Proceedings IEEE. IEEE, 2010: 1-9. 